\title{
Cardiac tamponade caused by an ectopic intrapericardial thymic carcinoma
}

\author{
Kai $Z \mathrm{Zh}^{1 *}$, Weijie Chen ${ }^{2 *}$, Sun Pan ${ }^{1}$, Chunsheng Wang ${ }^{1}$ \\ ${ }^{1}$ Department of Cardiac Surgery, Zhongshan Hospital, Fudan University, China \\ ${ }^{2}$ Department of Pathology, Zhongshan Hospital, Fudan University, China
}

A 69-year-old man was referred to our institute with slight dyspnea. Echocardiography showed a giant mass that occupied the pericardial cavity. 2-[18F]-fluoro-2-deoxy-D-glucose $\left({ }^{18} \mathrm{~F}-\mathrm{FDG}\right)$ positron emission tomography/magnetic resonance imaging (PET/MRI) revealed the mass $(7 \times 4 \mathrm{~cm})$ with low intensity on $\mathrm{T} 1$ weighted imaging, high intensity on T2 weighted imaging and diffusion weighted imaging $(b=800)$ (Suppl. Fig. S1A-C). PET/computed tomography (PET/CT) showed that the mass was FDG avid with the maximum standardized uptake value $\left(\mathrm{SUV}_{\max }\right)$ being 12.4 (Suppl. Fig. S1D). The patient refused surgery. Nine months later, he was delivered to our institute due to the presence of symptoms of cardiac tamponade. PET/ /CT showed an increased size $(10 \times 7 \mathrm{~cm})$ and FDG uptake $\left(\mathrm{SUV}_{\max }=17.7\right)$ of the mass (Fig. 1A).
$\mathrm{CT}$ angiography exhibited the presence of massive pericardial effusion (Fig. 1B).

Through a median sternotomy, the surgeon found that the mass was completely located in the pericardial cavity. The mass underwent radical resection, and the invaded superior vena cava and right atrium was reconstructed using a bovine pericardial patch under cardiopulmonary bypass (Fig. 1C, D). The patient felt symptomatic relief and was referred to oncologists to receive adjuvant therapy. Histopathology showed that the tumor consisted of epithelial cells (Fig. 1E, i) with positive immunostaining of CD5, CD117 and P63 (Fig. 1E, ii-iv). These supported a diagnosis of ectopic thymic carcinoma (type $\mathrm{C}$ ), which could have originated from aberrant thymic tissue left behind in pericardium during embryologic development of thymic gland. The patient has survived for over 14 months and further follow-up is ongoing.

Conflict of interest: None declared

Address for correspondence: Dr. Chunsheng Wang, Department of Cardiac Surgery, Zhongshan Hospital, Fudan University, 180 Fenglin Road, Shanghai 200032, China, tel: +8602164041990, e-mail: wangchunsheng@fudan.edu.cn

*Kai Zhu and Weijie Chen contribute equally to this work. 


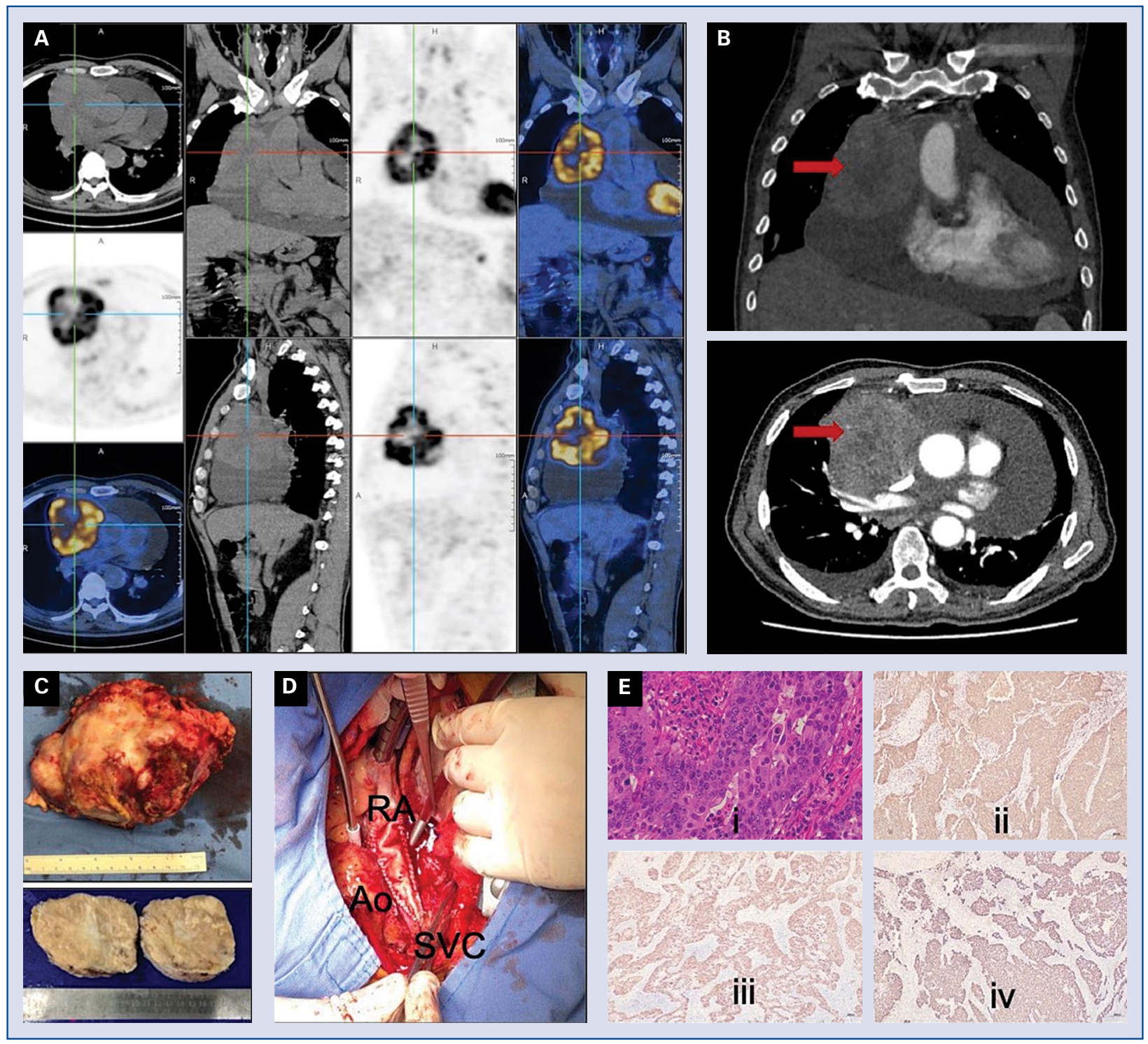

Figure 1. A. Positron emission tomography/computed tomography showed high avidity of the intrapericardial mass; B. Computed tomography angiography presented a close relationship between the mass (arrow) and surrounding structures. The mass was en bloc resected (C), and right atrium (RA) and superior vena cava (SVC) were reconstructed (D); E. Hematoxylin and eosin (i) and immunohistochemical staining for CD5 (ii), CD117 (iii) and P63 (iv) of the mass; Ao - ascending aorta. 